\title{
Higher-spin modes in a domain-wall universe
}

\section{Manuela Kulaxizi and Rakibur Rahman}

Université Libre de Bruxelles $\&$ International Solvay Institutes, ULB-Campus Plaine C.P. 231, B-1050 Bruxelles, Belgium

E-mail: manuela.kulaxizi@gmail.com, rakibur.rahman@ulb.ac.be

ABSTRACT: We find a consistent set of equations of motion and constraints for massive higher-spin fluctuations in a gravitational background, required of certain characteristic properties but more general than constant curvature space. Of particular interest among such geometries is a thick domain wall-a smooth version of the Randall-Sundrum metric. Apart from the graviton zero mode, the brane accommodates quasi-bound massive states of higher spin contingent on the bulk mass. We estimate the mass and lifetime of these higherspin resonances, which may appear as metastable dark matter in a braneworld universe.

Keywords: Higher Spin Symmetry, Large Extra Dimensions, Higher Spin Gravity

ARXiv EPRINT: 1409.1942 


\section{Contents}

1 Introduction $\quad 1$

2 Massive HS fields in a gravitational background 3

$\begin{array}{lll}3 & \text { The thick domain wall } & 6\end{array}$

4 Higher-spin fluctuations $\quad 8$

5 Concluding remarks $\quad 12$

$\begin{array}{ll}\text { A Some details of the involutive deformation } & 14\end{array}$

\section{Introduction}

Consistent interacting theories of higher-spin (HS) fields are difficult to construct. For massless fields, interactions are generically in tension with HS gauge invariance, and such pathologies lead to various no-go theorems in flat space [1-7]. Even the free propagation in non-trivial backgrounds may suffer from difficulties. Noticed long ago by Fierz and Pauli [8], the latter kind of problem shows up for massive fields at the level of equations of motion (EoMs) and constraints by rendering them mutually incompatible. A Lagrangian formulation takes care of this issue, but the resulting system is likely to propagate unphysical modes or allow propagation outside the light cone [9-17]. Appropriate non-minimal terms may come to the rescue and provide a consistent Lagrangian description of free massive HS fields in backgrounds with constant curvature [18-29].

Is it possible to describe consistently the free propagation of a massive field of arbitrary spin in spaces more general than the constant curvature ones? The answer is yes, at least at the level of EoMs and constraints, as we will show in this paper. The necessary conditions require only that the following irreducible Lorentz tensors, ${ }^{1}$ characteristic of the $D$-dimensional background metric $g_{\mu \nu}$, vanish:

$$
\begin{aligned}
X_{\mu \nu \rho}{ }^{\alpha \beta} & \equiv \nabla_{(\mu} W_{\nu}^{\alpha}{ }_{\rho)}^{\beta}-\left(\frac{2}{D+2}\right) g_{(\mu \nu} \nabla^{\sigma} W_{\rho)}\left(\alpha_{\sigma}^{\beta} \beta\right)=0, \\
Y_{\mu \nu \rho} & \equiv \nabla_{(\mu} R_{\nu \rho)}-\left(\frac{2}{D+2}\right) g_{(\mu \nu} \nabla_{\rho)} R=0, \\
Z_{\mu \nu \rho} & \equiv 2 \nabla_{[\rho} R_{\mu] \nu}+\left(\frac{1}{D-1}\right) g_{\nu[\rho} \nabla_{\mu]} R+(\mu \leftrightarrow \nu)=0,
\end{aligned}
$$

\footnotetext{
${ }^{1}$ The notation $\left(i_{1} \cdots i_{n}\right)$ means totally symmetric expression in all the indices $i_{1}, \ldots, i_{n}$ with the normalization factor $\frac{1}{n !}$. The totally antisymmetric expression $\left[i_{1} \cdots i_{n}\right]$ comes with the same normalization.
} 
where $W_{\mu \nu \rho \sigma}$ is the Weyl tensor, $R_{\mu \nu}$ the Ricci tensor, and $R$ the scalar curvature. In such a geometry, the consistent set of dynamical equations and constraints describing a probe totally symmetric spin- $s$ bosonic field $\varphi_{\mu_{1} \ldots \mu_{s}}$ will be given by

$$
\begin{gathered}
{\left[\nabla^{2}-M^{2}+\frac{2(s-1)(s+D-2)}{(D-1)(D+2)} \hat{R}\right] \varphi_{\mu_{1} \ldots \mu_{s}}+s(s-1) \hat{R}_{\left(\mu_{1}\right.}{ }^{\rho}{ }_{\mu_{2}}{ }^{\sigma} \varphi_{\left.\mu_{3} \ldots \mu_{s}\right) \rho \sigma}-s \hat{R}_{\rho\left(\mu_{1}\right.} \varphi^{\rho}{ }_{\left.\mu_{2} \ldots \mu_{s}\right)}=0} \\
\nabla \cdot \varphi_{\mu_{1} \ldots \mu_{s-1}} \equiv \nabla^{\mu_{s}} \varphi_{\mu_{1} \ldots \mu_{s}}=0 \\
\varphi_{\mu_{1} \ldots \mu_{s-2}}^{\prime} \equiv g^{\mu_{s-1} \mu_{s}} \varphi_{\mu_{1} \ldots \mu_{s}}=0
\end{gathered}
$$

where the quantity $\hat{R}_{\mu \nu \rho \sigma}$ is the Riemann tensor minus its constant trace part,

$$
\hat{R}_{\mu \nu \rho \sigma} \equiv R_{\mu \nu \rho \sigma}-\frac{2 \Lambda}{(D-1)(D-2)}\left(g_{\mu \rho} g_{\nu \sigma}-g_{\nu \rho} g_{\mu \sigma}\right),
$$

that conveniently parametrizes the deviation of the manifold under consideration from a constant curvature space of cosmological constant $\Lambda$, and $M$ is the mass in the latter. The assumptions include locality and that neither any vacuum expectation values, which possibly source the geometry, nor any other fluctuations show up at the linearized level. The existence of an underlying Lagrangian formulation, however, is not assumed.

A number of interesting geometries satisfy the conditions (1.1)-(1.3). Symmetric spaces have covariantly constant Riemann tensors: $\nabla_{\lambda} R_{\mu \nu \rho \sigma}=0$, and therefore qualify. Some coset spaces arising from supergravity and M-theory compactifications as well as some pp-wave backgrounds are of this kind. In particular, the well-known $\mathrm{AdS}_{5} \times \mathrm{S}^{5}$ geometry of string theory, or in fact any $\mathrm{AdS}_{p} \times \mathrm{S}^{q}$ even with unequal radii, is a symmetric space.

We will see that certain domain-wall (DW) geometries of phenomenological interest also fulfill the conditions (1.1)-(1.3). DW spacetimes in general arise naturally from a system of gravity plus scalar(s) with a potential. They play an important role in describing holographic renormalization group flows. Because there is an FLRW cosmology corresponding to every DW solution of a given model [30], these geometries are also interesting in the context of inflationary cosmology. Moreover, the Randall-Sundrum one-brane model [31] may find smooth generalizations through some DW solutions [32] (see also refs. $[33,34]$ and references therein, for example). Among the DW geometries that satisfy the conditions (1.1)-(1.3), there is indeed one that serves as a thick-brane realisation of the braneworld. The HS fluctuations on this geometry, governed by the eqs. (1.4)-(1.6), may therefore have phenomenologically interesting consequences.

The organization of this paper is as follows. The next section, which the reader may skip without loss of continuity, employs the "involutive deformation method" to derive the consistent set of EoMs and constraints (1.4)-(1.6) describing the free propagation of a massive spin- $s$ field in a gravitational background subject to the conditions (1.1)-(1.3). Some technical details of this section are relegated to appendix A. In section 3, we show that certain DW metrics with maximally symmetric slicings do fulfill the aforementioned criteria. In particular, there exists a smooth generalization of the Randall-Sundrum metric that also qualifies. We briefly recall the consequences the fluctuations of the latter geometry 
bring along, i.e., a localized graviton zero mode and a continuum of Kaluza-Klein modes on the thick brane. Section 4 considers HS fluctuations on top of this background. As the transverse traceless modes of the highest-spin field on the brane decouple completely from any other mode, the equivalent Schrödinger problem for them can be easily studied. Thankfully, normalizable HS zero modes are ruled out, but massive HS resonances on the brane are allowed. The mass and lifetime of these metastable HS states are estimated. We make some concluding remarks in section 5, notably that these HS resonances in a domain-wall universe may be so long lived as to qualify as dark matter candidates without contradicting the tests of the inverse-square law of gravity.

\section{Massive HS fields in a gravitational background}

A massive spin- $s$ bosonic field in flat space is customarily represented by a rank- $s$ symmetric traceless Lorentz tensor, say $\varphi_{\mu_{1} \ldots \mu_{s}}$. It satisfies the dynamical Klein-Gordon equation:

$$
I_{\mu_{1} \ldots \mu_{s}} \equiv\left(\partial^{2}-\mathfrak{m}^{2}\right) \varphi_{\mu_{1} \ldots \mu_{s}}=0,
$$

and is subject to the divergence and trace constraints:

$$
\begin{aligned}
J_{\mu_{1} \ldots \mu_{s-1}} & \equiv \partial \cdot \varphi_{\mu_{1} \ldots \mu_{s-1}}=0, \\
K_{\mu_{1} \ldots \mu_{s-2}} & \equiv \varphi_{\mu_{1} \ldots \mu_{s-2}}^{\prime}=0 .
\end{aligned}
$$

The divergence and trace constraints are crucial in the counting of propagating degrees of freedom $\mathfrak{D}$. In $D$ spacetime dimensions, it is given by

$$
\mathfrak{D}=2\left(\begin{array}{c}
D-4+s \\
s-1
\end{array}\right)+\left(\begin{array}{c}
D-4+s \\
s
\end{array}\right)
$$

which of course reduces to $2 s+1$ in $D=4$.

On the other hand, the mutual compatibility of the dynamical equation and constraints is indispensable for a consistent description. In other words, eqs. (2.1)-(2.3) can be viewed as an involutive system of differential equations [35], that fulfill the "gauge identities":

$$
\begin{aligned}
\mathcal{G}_{1, \mu_{1} \ldots \mu_{s-1}} & \equiv \partial \cdot I_{\mu_{1} \ldots \mu_{s-1}}-\left(\partial^{2}-\mathfrak{m}^{2}\right) J_{\mu_{1} \ldots \mu_{s-1}}=0 \\
\mathcal{G}_{2, \mu_{1} \ldots \mu_{s-2}} & \equiv I_{\mu_{1} \ldots \mu_{s-2}}^{\prime}-\left(\partial^{2}-\mathfrak{m}^{2}\right) K_{\mu_{1} \ldots \mu_{s-2}}=0 \\
\mathcal{G}_{3, \mu_{1} \ldots \mu_{s-3}} & \equiv J_{\mu_{1} \ldots \mu_{s-3}}^{\prime}-\partial \cdot K_{\mu_{1} \ldots \mu_{s-3}}=0
\end{aligned}
$$

thanks to the commutativity of ordinary derivatives. The above gauge identities however are not all independent, since the trace of $\mathcal{G}_{1, \mu_{1} \ldots \mu_{s-1}}$ can be expressed in terms of $\mathcal{G}_{2, \mu_{1} \ldots \mu_{s-2}}$ and $\mathcal{G}_{3, \mu_{1} \ldots \mu_{s-3}}$. In other words, there is a gauge identity for the gauge identities:

$$
\mathcal{H}_{\mu_{1} \ldots \mu_{s-3}} \equiv \mathcal{G}_{1, \mu_{1} \ldots \mu_{s-3}}^{\prime}-\partial \cdot \mathcal{G}_{2, \mu_{1} \ldots \mu_{s-3}}+\left(\partial^{2}-\mathfrak{m}^{2}\right) \mathcal{G}_{3, \mu_{1} \ldots \mu_{s-3}}=0
$$

From the point of view of an involutive system, the mutual compatibility of eqs. (2.1)-(2.3) is taken care of by the gauge identities [36]. It was shown long ago [37] that the degrees of freedom count is related to the "strength of the system". An explicit expression for $\mathfrak{D}$ is 
given in ref. [36] in terms of the number of equations $t_{k}$ and independent gauge identities $l_{k}$ of order $k$ in derivatives:

$$
\mathfrak{D}=\frac{1}{2} \sum_{k} k\left(t_{k}-l_{k}\right)
$$

Indeed, this formula reproduces the count (2.4) with the correct values of $t_{k}$ and $l_{k}$ :

$$
\begin{aligned}
& t_{k}=\delta_{k}^{2}\left(\begin{array}{c}
D+s-1 \\
s
\end{array}\right)+\delta_{k}^{1}\left(\begin{array}{c}
D+s-2 \\
s-1
\end{array}\right)+\delta_{k}^{0}\left(\begin{array}{c}
D+s-3 \\
s-2
\end{array}\right) \\
& l_{k}=\delta_{k}^{3}\left[\left(\begin{array}{c}
D+s-2 \\
s-1
\end{array}\right)-\left(\begin{array}{c}
D+s-4 \\
s-3
\end{array}\right)\right]+\delta_{k}^{2}\left(\begin{array}{c}
D+s-3 \\
s-2
\end{array}\right)+\delta_{k}^{1}\left(\begin{array}{c}
D+s-4 \\
s-3
\end{array}\right) .
\end{aligned}
$$

Consistency requires that any deformation of the flat-space free system (2.1)-(2.3) always fulfills the gauge identities. However, in a gravitational background, for example, the naïve covariantization $\partial_{\mu} \rightarrow \nabla_{\mu}$ of the flat-space system results in algebraic inconsistencies, since covariant derivatives no longer commute. Noticed already in ref. [8], such problems are in fact very generic for HS systems. For some special backgrounds, though, they may be cured by the addition of non-minimal terms. An explicit example of this appears below.

To consider the free propagation of a massive spin- $s$ particle in a gravitational background, we first deform the system (2.1)-(2.3) into the following:

$$
\begin{aligned}
I_{\mu_{1} \ldots \mu_{s}} & \equiv\left(\nabla^{2}-\mathfrak{m}^{2}\right) \varphi_{\mu_{1} \ldots \mu_{s}}+\Delta I_{\mu_{1} \ldots \mu_{s}}=0, \\
J_{\mu_{1} \ldots \mu_{s-1}} & \equiv \nabla \cdot \varphi_{\mu_{1} \ldots \mu_{s-1}}+\Delta J_{\mu_{1} \ldots \mu_{s-1}}=0, \\
K_{\mu_{1} \ldots \mu_{s-2}} & \equiv \varphi_{\mu_{1} \ldots \mu_{s-2}}^{\prime}+\Delta K_{\mu_{1} \ldots \mu_{s-2}}=0 .
\end{aligned}
$$

where the non-minimal deformations $\Delta I_{\mu_{1} \ldots \mu_{s}}, \Delta J_{\mu_{1} \ldots \mu_{s-1}}$ and $\Delta K_{\mu_{1} \ldots \mu_{s-2}}$ are linear in the field $\varphi_{\mu_{1} \ldots \mu_{s}}$, and contain at least one power of the curvature. They only contain lowerderivatives of the field lest unphysical modes should appear or causal propagation be lost. The involutive deformation method [36] consists of finding the deformations (2.12)-(2.14), for which there exists a deformed version of the relations (2.5)-(2.7), i.e.,

$$
\mathcal{G}_{i, \alpha_{1} \ldots \alpha_{s-i}} \equiv \mathcal{I}_{i, \alpha_{1} \ldots \alpha_{s-i}}^{\mu_{1} \ldots \mu_{s}} I_{\mu_{1} \ldots \mu_{s}}+\mathcal{J}_{i, \alpha_{1} \ldots \alpha_{s-i}}^{\mu_{1} \ldots \mu_{s-1}} J_{\mu_{1} \ldots \mu_{s-1}}+\mathcal{K}_{i, \alpha_{1} \ldots \alpha_{s-i}}^{\mu_{1} \ldots \mu_{s-2}} K_{\mu_{1} \ldots \mu_{s-2}}=0,
$$

where the operators $\mathcal{I}_{i}, \mathcal{J}_{i}, \mathcal{K}_{i}$ with $i=1,2,3$ are called the gauge identity generators. Again, they are minimal deformations of the free theory plus non-minimal corrections:

$$
\begin{aligned}
& \mathcal{I}_{i, \alpha_{1} \ldots \alpha_{s-i}}^{\mu_{1} \ldots \mu_{s}}=\delta_{i}^{1} \delta_{\alpha_{1} \ldots \alpha_{s-1}}^{\left(\mu_{1} \ldots \mu_{s-1}\right.} \nabla^{\left.\mu_{s}\right)}+\delta_{i}^{2} \delta_{\alpha_{1} \ldots \alpha_{s-2}}^{\left(\mu_{1} \ldots \mu_{s-2}\right.} g^{\left.\mu_{s-1} \mu_{s}\right)}+\Delta \mathcal{I}_{i, \alpha_{1} \ldots \alpha_{s-i}}^{\mu_{1} \ldots \mu_{s}}, \\
& \mathcal{J}_{i, \alpha_{1} \ldots \alpha_{s-i}}^{\mu_{1} \ldots \mu_{s-1}}=\delta_{i}^{1} \delta_{\alpha_{1} \ldots \alpha_{s-1}}^{\mu_{1} \ldots \mu_{s-1}}\left(-\nabla^{2}+\mathfrak{m}^{2}\right)+\delta_{i}^{3} \delta_{\alpha_{1} \ldots \alpha_{s-3}}^{\left(\mu_{1} \ldots \mu_{s-3}\right.} g^{\left.\mu_{s-2} \mu_{s-1}\right)}+\Delta \mathcal{J}_{i, \alpha_{1} \ldots \alpha_{s-i}}^{\mu_{1} \ldots \mu_{s-1}}, \\
& \mathcal{K}_{i, \alpha_{1} \ldots \alpha_{s-i}}^{\mu_{1} \ldots \mu_{s-2}}=\delta_{i}^{2} \delta_{\alpha_{1} \ldots \alpha_{s-2}}^{\mu_{1} \ldots \mu_{s-2}}\left(-\nabla^{2}+\mathfrak{m}^{2}\right)-\delta_{i}^{3} \delta_{\alpha_{1} \ldots \alpha_{s-3}}^{\left(\mu_{1} \ldots \mu_{s-3}\right.} \nabla^{\left.\mu_{s-2}\right)}+\Delta \mathcal{K}_{i, \alpha_{1} \ldots \alpha_{s-i}}^{\mu_{1} \ldots \mu_{s-2}}
\end{aligned}
$$

In appendix A, we have shown how the gauge identities (2.15) may be satisfied under the assumption of locality. It turns out the first gauge identity, $\mathcal{G}_{1, \alpha_{1} \ldots \alpha_{s-1}}=0$, can be fulfilled, with a free parameter $\alpha$, modulo that we set to zero certain anomalous terms containing derivatives of the curvature. These bad terms are given in eq. (A.7), and in order for them to vanish it is necessary that the gravitational background satisfy the 
conditions (1.1)-(1.3) for generic spin, namely $X_{\mu \nu \rho}{ }^{\alpha \beta}=0, Y_{\mu \nu \rho}=0$ and $Z_{\mu \nu \rho}=0$. The vanishing of the last term in eq. (A.7) further requires:

$$
\nabla_{\mu} R=0, \quad \text { or } \quad \alpha=\frac{2(s-1)(s+D-2)}{(D-1)(D+2)} .
$$

Now the freedom of the parameter $\alpha$ plays a crucial role. By choosing $\alpha$ to the above value, one may be able to do with a background of non-constant Ricci scalar: $\nabla_{\mu} R \neq 0$.

Under these conditions all the gauge identities can be fulfilled, with non-minimal corrections to the equations and gauge identity generators given by eqs. (A.4)-(A.6) and eqs. (A.8)-(A.14). These corrections in principle contain $\mathcal{O}\left(R^{2}\right)$-terms. However, they do not contribute at $\mathcal{O}\left(R^{2}\right)$, but only at $\mathcal{O}\left(R^{3}\right)$, in the gauge identities:

$$
\Delta \mathcal{I}_{i, \alpha_{1} \ldots \alpha_{s-i}}^{\mu_{1} \ldots \mu_{s}} \Delta I_{\mu_{1} \ldots \mu_{s}}+\Delta \mathcal{J}_{i, \alpha_{1} \ldots \alpha_{s-i}}^{\mu_{1} \ldots \mu_{s-1}} \Delta J_{\mu_{1} \ldots \mu_{s-1}}+\Delta \mathcal{K}_{i, \alpha_{1} \ldots \alpha_{s-i}}^{\mu_{1} \ldots \mu_{s-2}} \Delta K_{\mu_{1} \ldots \mu_{s-2}}=\mathcal{O}\left(R^{3}\right) .
$$

This means that in the deformations (2.12)-(2.14) all the the higher-curvature terms can be consistently set to zero. The resulting system has undeformed divergence and trace:

$$
\begin{aligned}
{\left[\nabla^{2}-\mathfrak{m}^{2}+\alpha R\right] \varphi_{\mu_{1} \ldots \mu_{s}}+s(s-1) R_{\left(\mu_{1}\right.}{ }^{\rho}{ }_{\mu_{2}}{ }^{\sigma} \varphi_{\left.\mu_{3} \ldots \mu_{s}\right) \rho \sigma}-s R_{\rho\left(\mu_{1}\right.} \varphi^{\rho}{ }_{\left.\mu_{2} \ldots \mu_{s}\right)} } & =0 \\
\nabla \cdot \varphi_{\mu_{1} \ldots \mu_{s-1}} & =0 \\
\varphi_{\mu_{1} \ldots \mu_{s-2}}^{\prime} & =0
\end{aligned}
$$

This system is consistent, under the conditions (1.1)-(1.3) and (2.19), up to all orders in the curvature. Note that the addition of $\mathcal{O}\left(R^{2}\right)$ terms, which is inessential for consistency, may require further conditions. For a background with a non-constant Ricci scalar, $\alpha$ must be set to the value of eq. (2.19). The EoMs and constraints (1.4)-(1.6) then follow from incorporating the constant trace part of the curvature tensor into the mass term.

One still needs to check that there exists a deformed counterpart of the identity (2.8). A straightforward computation gives

$$
\begin{aligned}
& \mathcal{G}_{1, \mu_{1} \ldots \mu_{s-3}}^{\prime}-\nabla \cdot \mathcal{G}_{2, \mu_{1} \ldots \mu_{s-3}}+\left(\nabla^{2}-\mathfrak{m}^{2}\right) \mathcal{G}_{3, \mu_{1} \ldots \mu_{s-3}} \\
& =-(s-3)(s-4) R_{\left(\mu_{1}{ }^{\rho}{ }{ }_{2}{ }^{\sigma} \mathcal{G}_{\left.3, \mu_{3} \ldots \mu_{s-3}\right) \rho \sigma}+(s-3) R^{\rho}{ }_{\left(\mu_{1}\right.} \mathcal{G}_{\left.3, \mu_{2} \ldots \mu_{s-3}\right) \rho}-\alpha R \mathcal{G}_{3, \mu_{1} \ldots \mu_{s-3}}\right.} \\
& \quad+\left[\alpha-\frac{2(s-3)(s+D-4)}{(D-1)(D+2)}\right]\left(\nabla^{\rho} R\right) K_{\mu_{1} \ldots \mu_{s-3} \rho}+\Delta \mathcal{K}_{1, \mu_{1} \ldots \mu_{s-3}}^{\prime \alpha_{1} \ldots \alpha_{s-2}} K_{\alpha_{1} \ldots \alpha_{s-2}} .
\end{aligned}
$$

Therefore, $\mathcal{G}_{1, \mu_{1} \ldots \mu_{s-3}}^{\prime}$ can be expressed completely in terms of $\mathcal{G}_{2, \mu_{1} \ldots \mu_{s-2}}$ and $\mathcal{G}_{3, \mu_{1} \ldots \mu_{s-3}}$ provided that the last line in eq. (2.24) vanishes. For $\nabla_{\mu} R=0$, the latter condition is automatic. When $\nabla_{\mu} R \neq 0$, there are two possibilities: one is to start with a field whose trace is vanishing identically rather than just as an on-shell condition $[36,38]$. In this case, $K_{\mu_{1} \ldots \mu_{s-2}}$ would never appear in the system and its reduced number of gauge identities. By so doing, one would demand that the trace always remain zero, even in the presence of interactions. ${ }^{2}$ Another possibility is to view our original system (2.1)-(2.3) as

\footnotetext{
${ }^{2}$ Such a requirement may have non-trivial consequences in a possible Lagrangian formulation of the system. We thank I. L. Buchbinder and Y. M. Zinoviev for stressing out this point.
} 
the zero-trace gauge fixing of a system of symmetric rank- $s$ field with a Weyl symmetry: $\delta \varphi_{\mu_{1} \ldots \mu_{s}}=g_{\left(\mu_{1} \mu_{2}\right.} \lambda_{\left.\mu_{3} \ldots \mu_{s}\right)}$. Now the freedom of the rank- $(s-2)$ parameter $\lambda_{\mu_{1} \ldots \mu_{s-2}}$ allows one to choose the trace to vanish even at the interaction level. ${ }^{3}$ The massless counterpart of such a system is well known in the literature as Conformal Higher Spin [39-41] (See also refs. [42-56] for recent discussions).

\section{The thick domain wall}

Let us consider the following domain wall metric in $D=d+1$ dimensions

$$
d s^{2}=d y^{2}+e^{2 f(y)}\left[-\left(1-k r^{2}\right) d t^{2}+\frac{d r^{2}}{1-k r^{2}}+r^{2} d \Omega_{d-2}\right], \quad-\infty<y<+\infty,
$$

where $k=(-1,0,+1)$ correspond respectively to $d$-dimensional AdS, flat and dS slicings. We would like to see if such a geometry can possibly satisfy the conditions (1.1)-(1.3). Because the metric (3.1) is conformally flat, $X_{\mu \nu \rho}{ }^{\alpha \beta}=0$ automatically. It turns out that $Z_{\mu \nu \rho}=0$ for any $f(y)$ as well. The only non-trivial condition on the metric is imposed by the vanishing of the tensor $Y_{\mu \nu \rho}$; it requires $f(y)$ to satisfy the following differential equation:

$$
f^{\prime \prime \prime}-2 f^{\prime} f^{\prime \prime}-4 k e^{-2 f} f^{\prime}=0 .
$$

The generic solution of this equation is given in terms of Jacobi elliptic functions:

$$
f(y)=-\ln \left[a \operatorname{sn}\left(b+\frac{y}{l}, k a^{2} l^{2}\right)\right] .
$$

where $a, b$ and $l \neq 0$ are constants.

For $k=0$, the metric (3.1) boils down to one with $d$-dimensional Poincaré invariance:

$$
d s^{2}=d y^{2}+e^{2 f(y)} \eta^{i j} d x_{i} d x_{j}
$$

where $\eta^{i j}$ is the flat metric with $i, j=0,1, \ldots, d-1$. The general solution (3.3), on the other hand, reduces to

$$
f(y)=-\ln \left[a \cosh \left(b+\frac{y}{l}\right)\right]
$$

which of course obeys the differential equation

$$
f^{\prime \prime}=f^{\prime 2}-l^{-2}
$$

The solution (3.5) satisfies the null energy condition with real $b$ and $l$, since

$$
T_{t}^{t}-T_{y}^{y}=-\frac{3}{l^{2}} \operatorname{sech}^{2}\left(b+\frac{y}{l}\right) \leq 0
$$

Note that pure $\operatorname{AdS}_{d+1}$ of radius $l=\sqrt{-d(d-1) / 2 \Lambda}$ solves eq. (3.6) with $f^{\prime}=l^{-1}$ and $f^{\prime \prime}=0$. For more generic solutions (3.5), therefore, the quantity $f^{\prime \prime}$ will parametrize

\footnotetext{
${ }^{3}$ We are thankful to M. Taronna for bringing this possibility to our attention.
} 
the deviation from AdS space. In other words, the "hatted" Riemann tensor $\hat{R}_{\mu \nu \rho \sigma}$ defined in eq. (1.7) will be proportional to $f^{\prime \prime}$. Indeed, its non-zero content is given by

$$
\hat{R}_{j}^{i}=-(d+1) \delta_{j}^{i} f^{\prime \prime}, \quad \hat{R}_{y}^{y}=-2 d f^{\prime \prime}, \quad \hat{R}=-d(d+3) f^{\prime \prime} .
$$

The thick-brane solution we will be interested in corresponds to a simple choice of parameters: $a=1$ and $b=0$ in eqs. (3.4)-(3.5). This gives

$$
f(y)=-\ln \cosh \left(\frac{y}{l}\right),
$$

which represents a smooth generalization of the Randall-Sundrum metric [31], the thickness of the brane being $\mathcal{O}(l)$. This particular thick-brane generalization has already been studied in refs. $[57,58]$. Note that the metric (3.9) is conformally flat with a non-constant Ricci scalar, and does not asymptote to AdS space.

We will consider massive HS fluctuations in this geometry. Although, in the context of braneworlds, the massless case has been studied by some authors [59-61], no study of the massive ones seems to be present. But first let us discuss briefly the graviton fluctuations.

Graviton fluctuations. Universal aspects of graviton fluctuations in conformally flat backgrounds preserving $d$-dimensional Poincaré invariance have been extensively studied in the literature. Here we follow ref. [62]. From the $d$-dimensional point of view, graviton fluctuations of the form $h_{i j}(x, y)=\psi(y) \epsilon_{i j} e^{i q \cdot x}$ will obey the following equation in the transverse traceless gauge:

$$
\left[\partial_{y}^{2}+(d-4) f^{\prime} \partial_{y}-e^{-2 f} q^{2}-2 f^{\prime \prime}-2(d-2) f^{\prime 2}\right] \psi(y)=0
$$

which can be derived from the Einstein equations in the bulk. Note that in the next section we are going to present a generalization (4.3) of this equation for the transverse traceless modes of a fluctuation of arbitrary spin and mass. For a massless graviton in the bulk, with $M^{2} l^{2}=-2$ [63-65], indeed the general equation reduces to the above one.

The existence of normalizable $d$-dimensional modes is connected with the asymptotic behavior of the potential of the equivalent Schrödinger problem. It turns out there are no normalizable negative energy graviton modes (with $-q^{2}<0$ ). For $-q^{2}=0$, there is a normalizable mode $[57,58]$ given by

$$
\psi_{0}(y)=\sqrt{\frac{3}{4 l}} \operatorname{sech}^{2}\left(\frac{y}{l}\right),
$$

which is identified as the localized massless graviton on the brane.

There are no massive graviton bound states nor any resonances [57, 58], but a continuum of Kaluza-Klein modes for all $-q^{2}>0$, as they usually appear [31, 62]. This can be shown, for example, from the generalized case of the next section. These Kaluza-Klein modes will alter the behavior of gravity at length scale $\mathcal{O}(l)$ [31]. In particular, Newton's inverse square law will get modified, and this poses an upper bound on $l$ from table-top experiments [66]. The bound turns out to be $l \lesssim 10^{-4} \mathrm{~m}$. 


\section{Higher-spin fluctuations}

In conformally flat backgrounds, in general, the HS dynamical equation (1.4) reduces to

$$
\left[\nabla^{2}-M^{2}+\beta \hat{R}\right] \varphi_{\mu_{1} \ldots \mu_{s}}-\frac{s(2 s+d-3)}{d-1} \hat{R}_{\left(\mu_{1}\right.}^{\rho} \varphi_{\left.\mu_{2} \ldots \mu_{s}\right) \rho}+\frac{s(s-1)}{d-1} \hat{R}^{\rho \sigma} g_{\left(\mu_{1} \mu_{2}\right.} \varphi_{\left.\mu_{3} \ldots \mu_{s}\right) \rho \sigma}=0,
$$

where $\beta=\frac{(s-1)\left[s(3 d+1)+2(d-1)^{2}\right]}{d(d-1)(d+3)}$. Along with the divergence and trace constraints, this equation is suitable for describing small fluctuations of HS fields in the domain-wall geometries listed above. Let us consider higher-spin fluctuations of the form:

$$
\varphi_{\mu_{1} \ldots \mu_{s}}(x, y)=\int \frac{d^{d} q}{(2 \pi)^{d}} \tilde{\varphi}_{\mu_{1} \ldots \mu_{s}}(q, y) e^{i q \cdot x} .
$$

on the flat DW background (3.4). The component of $\tilde{\varphi}_{\mu_{1} \ldots \mu_{s}}(q, y)$ with $r$ indices in the $y$-direction $(0 \leq r \leq s)$ will appear as the Fourier transform of a spin- $(s-r)$ field to an observer on the brane. The transverse traceless modes of the spin- $s$ field $(r=0)$ decouple completely from the other fields at the level of EoMs and constraints; they satisfy

$$
\left[\partial_{y}^{2}+(d-2 s) f^{\prime} \partial_{y}-e^{-2 f} q^{2}-M^{2}+s(s-d-1)-2(d-1)(s-1) f^{\prime \prime}\right] \tilde{\varphi}(y)=0,
$$

where we have suppressed the indices and $q$-dependence of $\tilde{\varphi}$. For $f(y)$ given by eq. (3.9), the above equation can be brought into the Schrödinger form through the following redefinitions of coordinate and variable:

$$
u=\sinh y, \quad \Psi(u)=\left[1+u^{2}\right]^{\frac{2 s-d+1}{4}} \tilde{\varphi}(u),
$$

where we have set $l=1$ for simplicity. Thus one arrives at

$$
\left[-\partial_{u}^{2}+V(u)\right] \Psi(u)=-q^{2} \Psi(u),
$$

where the potential $V(u)$ is of the form

$$
V(u)=\frac{A u^{2}}{\left(1+u^{2}\right)^{2}}+\frac{M^{2}-B}{1+u^{2}},
$$

with the coefficients $A$ and $B$ depending on the spin and dimensionality as follows:

$$
\begin{aligned}
& A=\frac{1}{4}(2 s+d-3)^{2}-1, \\
& B=A-s-\frac{1}{4}\left(d^{2}-1\right)<A .
\end{aligned}
$$

Note that for all $s \geq 1$ and $d \geq 3$ we have $A \geq 0$, and $M^{2} \geq B-A$. The latter fact follows from the generalization of the BF bound [67] on the AdS mass for $s \geq 1$ [63-65]:

$$
M^{2} \geq s^{2}+s(d-5)-2(d-2) .
$$

The potential $V(u)$ is symmetric under reflection, $u \rightarrow-u$, and vanishes as $u \rightarrow \pm \infty$. It has a distinct volcano shape for the following range of the bulk mass:

$$
B-A<M^{2}<B+A .
$$




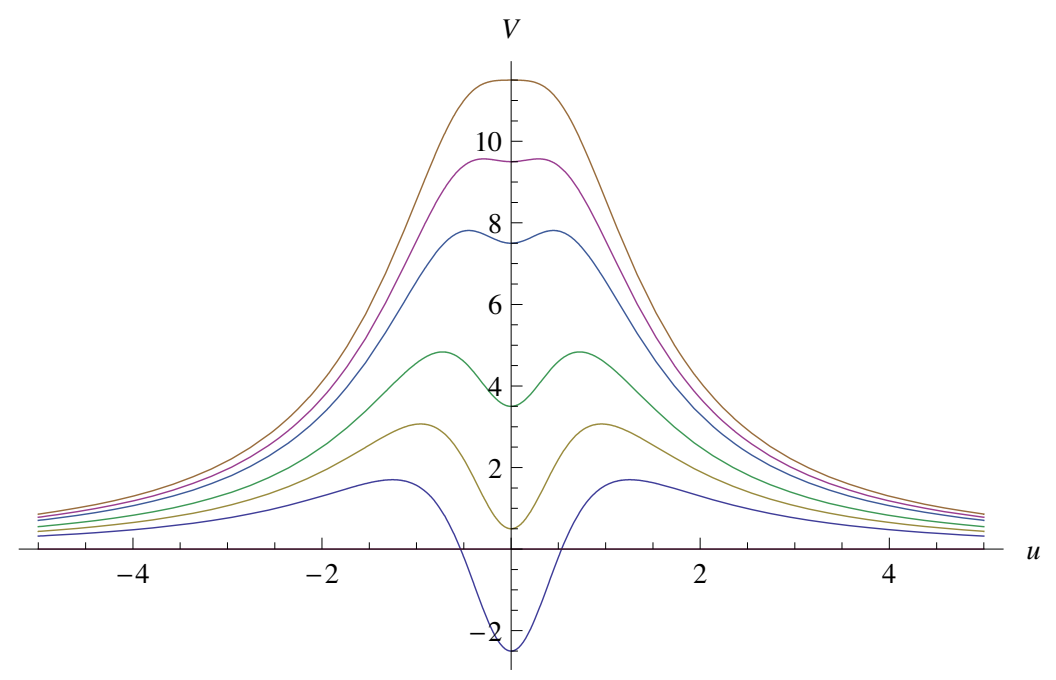

Figure 1. The potential $V(u)$ for a particle of spin $s=3$ in $d=4$ dimensions and values of the bulk mass $M^{2}=2,5,8,12,14,16$.

The local minimum appears at $u=0$, and the two maxima at $u= \pm \sqrt{\frac{A+B-M^{2}}{A-B+M^{2}}}$. The crater goes above zero at $M^{2}=B$. The minimum and maxima disappear for $M^{2} \geq B+A$, in which case a bell-shaped potential shows up (see figure1).

To study the spectrum on the domain wall, let us first note that $q^{2}$ is to be interpreted as the momentum squared of the $d$-dimensional fields. As a consistency check one needs to ensure that normalizable tachyonic modes do not exist. Indeed, it is easy to see that eq. (4.5) does not admit non-trivial solutions for $-q^{2}<0$ that vanishes at infinity. Below we discuss the (im)possibility of having localized massless and massive HS modes.

Zero modes and absence thereof. Massless modes correspond to $-q^{2}=0$, for which the solution of eq. (4.5) is given in terms of associated Legendre polynomials for generic values of the parameters:

$$
\Psi(u)=\sqrt{1+u^{2}}\left[c_{1} P_{\nu}^{\mu}(i u)+c_{2} Q_{\nu}^{\mu}(i u)\right],
$$

where

$$
\nu=\sqrt{M^{2}+\left(\frac{d}{2}\right)^{2}+s}-\frac{1}{2}, \quad \mu=s+\frac{1}{2}(d-3) .
$$

There exist no normalizable solutions for generic $\mu, \nu$. But when $\nu=\mu-n-1$, with $n \in \mathbb{N}$, the associated Legendre polynomials do not constitute a set of independent solutions: one solution is of hypergeometric type, while the other is given by $\Psi(u)=\left(1+u^{2}\right)^{\frac{1-\mu}{2}} p_{n}(u)$, where $p_{n}(u)$ is a polynomial of degree $n$, which is even(odd) for even(odd) $n$. In this case, the asymptotic behavior of the wave function is $\Psi(u) \sim u^{-\nu}$. For $s \geq 1$ and $d \geq 3$, both $\nu$ and $\mu$ are positive, and normalizable higher-spin zero modes seem to show up.

Upon inclusion of the coupling to dynamical gravity in the bulk, this would suggest the existence of gravitationally coupled massless higher-spin fields on the flat domain wall. 
This is however in direct contradiction with old $[1,4,5]$ and new [7] no-go theorems, which can actually be combined to completely rule out any gravitational coupling of massless higher spins in flat space [68]. The resolution of the puzzle lies in the values of the bulk mass yielding the zero modes. The relation between $\nu$ and $\mu$ gives:

$$
M^{2}=(n-s+2)(n-s-d+2)-s, \quad n=0,1, \ldots, \frac{1}{2}(2 s+d-4) .
$$

But these are precisely the points where the field is (partially) massless in $\operatorname{AdS}_{d+1}$ [69]. The points $n \geq 1$ are excluded simply because they fall outside the unitarity region (4.9). Neither is the value $n=0$ allowed. To see this, let us note the system (1.4)-(1.6) can be viewed as a deformation around AdS. Now $n=0$ corresponds to a massless field in AdS. However, the associated gauge invariance will be lost in the more generic manifold under consideration. In other words, the massless case has to be excluded from the beginning for non-constant curvature spaces. Thus there are no contradictions with the no-go theorems. In the model [31], an apparent contradiction of the similar kind was seen to arise [61].

Massive quasi-bound states. Let us now consider massive modes. When the bulk mass lies within the region (4.10), the potential acquires a volcano shape and quasi-bound states/resonances may show up. ${ }^{4}$ For an analytic study of the quasi-bound states, let us first rescale the coordinate as:

$$
z \equiv \sqrt{2}\left(A+B-M^{2}\right)^{\frac{1}{4}} u .
$$

Then a Taylor expansion of the potential (4.6) around $z=0$ reduces eq. (4.5) to the anharmonic oscillator problem:

$$
\left[-\partial_{z}^{2}+\frac{1}{4} z^{2}+\sum_{p=2}^{\infty} \frac{\left(p A+B-M^{2}\right) z^{2 p}}{\left(-2 \sqrt{A+B-M^{2}}\right)^{p+1}}\right] \Psi(z)=E \Psi(z)
$$

where the energy $E$ is related to $-q^{2}$ as follows:

$$
E=\frac{-q^{2}-M^{2}+B}{2 \sqrt{A+B-M^{2}}}
$$

For $A \gg 1$ and $M^{2}$ not very close to the upper bound $A+B$, the anharmonic terms can be treated as perturbation. As an approximation, we will reduce the problem to that of ref. [70] by keeping only the first term, $\frac{1}{4} \lambda z^{4}$, where the perturbation parameter is

$$
\lambda=-\frac{\left(2 A+B-M^{2}\right)}{2\left(A+B-M^{2}\right)^{3 / 2}}<0 .
$$

The associated boundary condition $\lim _{|z| \rightarrow \infty} \Psi(z)=0$ will select a discrete set of energy eigenvalues, which are complex [70]. They correspond to metastable states for $|\lambda| \ll 1$. The approximation of our original problem to that of ref. [70] will make sense if we restrict

\footnotetext{
${ }^{4}$ Bound states are excluded because for $-q^{2} \neq 0$ the wave function becomes oscillatory as $u \rightarrow \pm \infty$.
} 


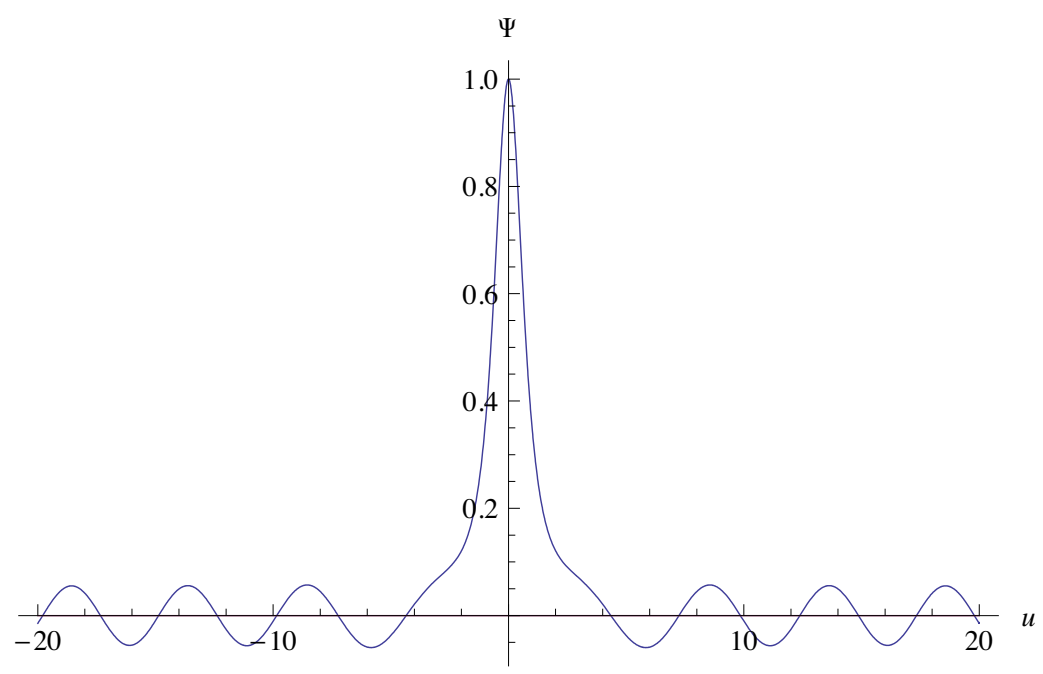

Figure 2. A spin- 4 resonance at $m^{2}=1.687$ in $d=4$, for bulk mass $M^{2}=10$. The amplitude of oscillations at large $u$ is approximately 0.06 with the wave function normalized to unity at the center: $\Psi(u=0)=1$.

ourselves to such eigenfunctions as are peaked at $z=0$, and have a much lower amplitude away from the origin. Therefore, we will consider only the ground state of the anharmonic oscillator, for which the energy is given by [70]:

$$
\operatorname{Re}(E) \approx \frac{1}{2}-\frac{3|\lambda|}{4}, \quad \operatorname{Im}(E) \approx-\sqrt{\frac{8}{\pi|\lambda|}} \exp \left(-\frac{1}{3|\lambda|}\right) .
$$

Note that $\operatorname{Im}(E)$ is exponentially small. In view of eq. (4.16), $-q^{2}$ will also be complex:

$$
-q^{2}=\left(m-\frac{i}{2} \Gamma\right)^{2}
$$

where $m$ is the mass and $\Gamma$ is the width of the metastable state, with $\Gamma \ll m$. Comparing eqs. (4.16), (4.18) and (4.19), one finds that the mass is given by

$$
m^{2} \approx M^{2}-B+\sqrt{A+B-M^{2}}-\frac{3}{4}\left(\frac{2 A+B-M^{2}}{A+B-M^{2}}\right)
$$

while the lifetime, $\tau=1 / \Gamma$, is

$$
\tau \approx\left[\frac{\pi\left(2 A+B-M^{2}\right)\left(M^{2}-B+\sqrt{A+B-M^{2}}\right)}{32\left(A+B-M^{2}\right)^{5 / 2}}\right]^{1 / 2} \exp \left[\frac{2\left(A+B-M^{2}\right)^{3 / 2}}{3\left(2 A+B-M^{2}\right)}\right] .
$$

One may resort to numerics to see if highly-peaked resonances are indeed present. The Schrödinger equation (4.5), with the boundary conditions $\Psi(u=0)=1$ and $\Psi^{\prime}(u=0)=0$, can be solved numerically. The amplitude at $u=0$ is chosen to be unity. We then scan the 


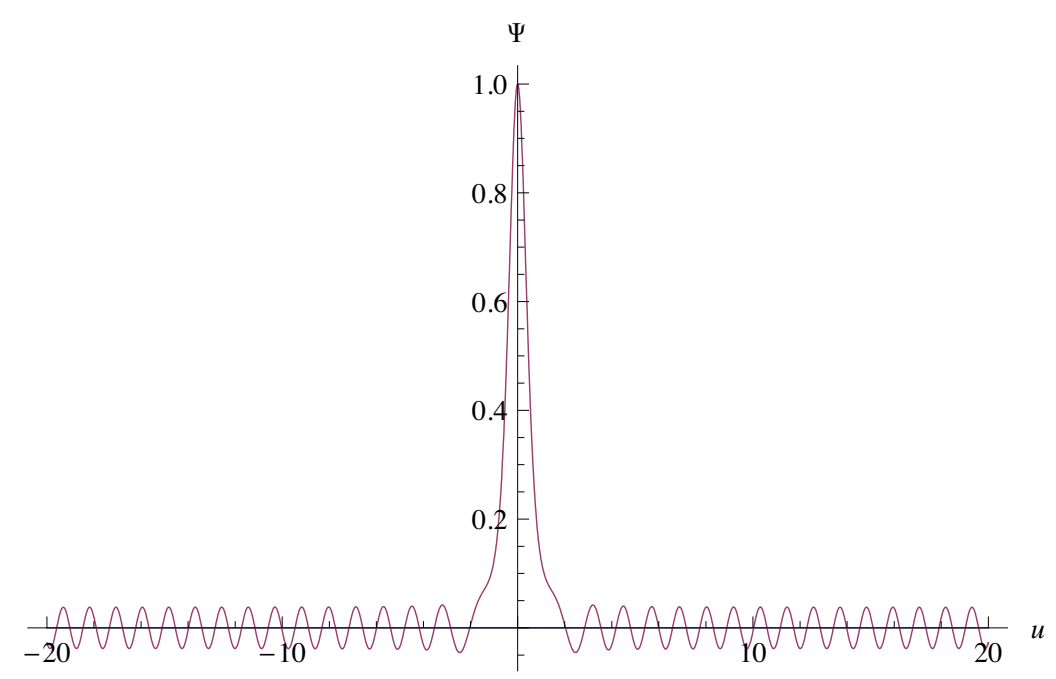

Figure 3. A spin-10 resonance at $m^{2}=31.900$ in $d=4$, for bulk mass $M^{2}=120$. The amplitude of oscillations at large $u$ is approximately 0.04 with the wave function normalized to unity at the center: $\Psi(u=0)=1$.

solutions for different $m^{2}$ until we find a solution for which the amplitude of oscillations at infinity is much smaller than unity. Given a value of the bulk mass in the range (4.10), this procedure gives a single resonant mode at $m^{2}=m^{2}\left(M^{2}\right)$ for each $s \geq 2$ in $d=4$.

The numerical result for the mass matches well with the value (4.20), and therefore to the ground state energy eigenvalue (4.18). Figures 2 and 3 show the resonant wave function $\Psi(u)$ for specific values of the bulk mass and spin in $d=4$ dimensions. For excited-state eigenvalues of the anharmonic oscillator, the wave function around $u=0$ oscillates with an amplitude comparable to that outside the volcano, and thus the existence of a resonance cannot be established.

\section{Concluding remarks}

In this paper, we have written down a consistent set of EoMs and constraints for a free massive HS field propagating in a gravitational background. The required characteristics of the geometry ${ }^{5}$ allow for spaces of non-constant curvature. In particular, we found a thick-brane realization of the Randall-Sundrum braneworld that admits consistent free propagation of massive HS fluctuations. The brane is seen to accommodate not only the graviton but also massive higher-spin resonances, whose mass and lifetime are estimated.

May these HS modes appear as dark matter in a braneworld universe? The idea of higher-spin dark matter has been explored in ref. [80]. It is natural for massive HS particles not to couple directly to the Standard Model, and so they are appealing as realistic dark matter candidates. To qualify as stable dark matter, their lifetime has to exceed the age

\footnotetext{
${ }^{5}$ Curiously, the consistency of the Lagrangian dynamics of spinning particles in various dimensions imposes similar restrictions on the backgrounds [71-79].
} 
of the universe: $\tau \gtrsim 10^{10}$ years $\sim 10^{26} \mathrm{~m}$. To see if this is possible in our setup, let us choose for simplicity the typical value $M^{2}=B$ of the bulk mass. One can reintroduce the parameter $l$ to rewrite eqs. (4.20) and (4.21) as

$$
m^{2} l^{2} \approx \sqrt{A}-\frac{3}{2}, \quad \frac{\tau}{l} \approx \sqrt{\frac{\pi}{16 A}} \exp \left(\frac{\sqrt{A}}{3}\right) .
$$

As already mentioned in section 3 , tests of gravity set $l \lesssim 10^{-4} \mathrm{~m}[66]$. This means $\frac{\tau}{l} \gtrsim 10^{30}$, which corresponds to a relatively stable dark matter particle with spin $s \gtrsim 230$. The mass turns out to be interesting from a phenomenological point is view: $m \gtrsim 1 \mathrm{TeV}$.

We expect these HS particles to couple to gravity like ordinary matter, i.e., to obey the principle of equivalence. In principle, one can go beyond the free-propagation level and consider gravitational coupling of the HS fields in the bulk. Because the fields are massive, their interactions with gravity do not suffer from any immediate issues originating from gauge invariance, unlike the massless [1-7] and partially massless [81-87] cases. This is however beyond the scope of our present work. Their interpretation as dark matter necessarily calls for such a study, though. This will be very important in understanding the details of such dark matter candidates and their possible role in the cosmological evolution of our universe.

Our paper was the first step in trying to describe the propagation of HS fields in DW backgrounds. For simplicity, we did not consider their coupling to the profile of the scalar field(s) that may source the geometry. It is possible that the inclusion of the scalar profile allow for more geometries of phenomenological interest. Another interesting direction to pursue is the case of non-zero $k$, i.e., (A)dS slicings. This may admit some asymptotically AdS geometries that could be studied holographically. We leave this as future work.

\section{Acknowledgments}

We would like to thank R. Argurio, L. Calibbi, A. Campoleoni, A. Parnachev, M. Porrati, S. Rychkov, E. D. Skvortsov, M. Taronna and A. Waldron for useful discussions. The work of MK was supported in part by the ERC Advanced Grant "SyDuGraM", by IISN-Belgium (convention 4.4514.08) and by the "Communauté Française de Belgique" through the ARC program. She gratefully acknowledges the National Science Foundation Grant No. PHYS1066293 and the hospitality of the Aspen Center for Physics, as well as support from the Simons Center for Geometry and Physics, Stony Brook University during the 2014 Simons Summer Workshop at which some of the research for this paper was performed. MK is also thankful to the Orthodox Academy of Crete at Kolymbari for hospitality during the "Quantum Field Theory, String Theory and Condensed Matter Physics" meeting. RR is a Postdoctoral Fellow of the Fonds de la Recherche Scientifique-FNRS. His work is partially supported by IISN-Belgium (conventions 4.4511.06 and 4.4514.08) and by the "Communauté Française de Belgique" through the ARC program. He is grateful to the Centre of Theoretical Physics at Tomsk State Pedagogical University for its kind hospitality during the conference QFTG'14, where a part of this work was done. 


\section{A Some details of the involutive deformation}

Our convention for the covariant derivative is: $\left[\nabla_{\mu}, \nabla_{\nu}\right] V^{\rho}=R_{\sigma \mu \nu}^{\rho} V^{\sigma}$. One can write down the various contributions to the quantity $\mathcal{G}_{i, \alpha_{1} \ldots \alpha_{s-i}}$, defined in eq. (2.15), as:

$$
\begin{aligned}
\mathcal{G}_{i, \alpha_{1} \ldots \alpha_{s-i}}= & \Delta \mathcal{I}_{i, \alpha_{1} \ldots \alpha_{s-i}}^{\mu_{1} \ldots \mu_{s}} I_{\mu_{1} \ldots \mu_{s}}+\Delta \mathcal{J}_{i, \alpha_{1} \ldots \alpha_{s-i}}^{\mu_{1} \ldots \mu_{s-1}} J_{\mu_{1} \ldots \mu_{s-1}}+\Delta \mathcal{K}_{i, \alpha_{1} \ldots \alpha_{s-i}}^{\mu_{1} \ldots \mu_{s-2}} K_{\mu_{1} \ldots \mu_{s-2}} \\
& +\delta_{i}^{1}\left[-\mathcal{A}_{\alpha_{1} \ldots \alpha_{s-1}}+\nabla \cdot \Delta I_{\alpha_{1} \ldots \alpha_{s-1}}-\left(\nabla^{2}-\mathfrak{m}^{2}\right) \Delta J_{\alpha_{1} \ldots \alpha_{s-1}}\right] \\
& +\delta_{i}^{2}\left[\Delta I_{\alpha_{1} \ldots \alpha_{s-2}}^{\prime}-\left(\nabla^{2}-\mathfrak{m}^{2}\right) \Delta K_{\alpha_{1} \ldots \alpha_{s-2}}\right] \\
& +\delta_{i}^{3}\left[\Delta J_{\alpha_{1} \ldots \alpha_{s-3}}^{\prime}-\nabla \cdot \Delta K_{\alpha_{1} \ldots \alpha_{s-3}}\right]
\end{aligned}
$$

where $\mathcal{A}_{\alpha_{1} \ldots \alpha_{s-1}}$ is the sole contribution from the minimal theory:

$$
\mathcal{A}_{\alpha_{1} \ldots \alpha_{s-1}} \equiv\left[\nabla^{2}, \nabla^{\mu}\right] \varphi_{\mu \alpha_{1} \ldots \alpha_{s-1}} \neq 0
$$

which calls for non-minimal corrections to the system (2.12)-(2.14) under consideration. This is a 1-derivative term linear in the curvature. One can use Leibniz rule to extract out of it various other pieces present in the correct gauge identity (A.1), up to some anomalous terms. Locality admits a unique result up to one free parameter $\alpha$ :

$$
\begin{aligned}
\mathcal{A}_{\alpha_{1} \ldots \alpha_{s-1}}= & \nabla \cdot \Delta \tilde{I}_{\alpha_{1} \ldots \alpha_{s-1}}+\Delta \tilde{\mathcal{J}}_{1, \alpha_{1} \ldots \alpha_{s-1}}^{\mu_{1} \ldots \mu_{s-1}} \nabla \cdot \varphi_{\mu_{1} \ldots \mu_{s-1}}+\Delta \tilde{\mathcal{K}}_{1, \alpha_{1} \ldots \alpha_{s-1}}^{\mu_{1} \ldots \mu_{s-2}} \varphi_{\mu_{1} \ldots \mu_{s-2}}^{\prime} \\
& +\mathcal{B}_{\alpha_{1} \ldots \alpha_{s-1}},
\end{aligned}
$$

where the first-order correction $\Delta \tilde{I}_{\alpha_{1} \ldots \alpha_{s}}$ to the dynamical equation is given by

$$
\Delta \tilde{I}_{\alpha_{1} \ldots \alpha_{s}}=s(s-1) R_{\left(\alpha_{1}\right.}{ }^{\rho} \alpha_{2}{ }^{\sigma} \varphi_{\left.\alpha_{3} \ldots \alpha_{s}\right) \rho \sigma}-s R_{\rho\left(\alpha_{1}\right.} \varphi^{\rho}{ }_{\left.\alpha_{2} \ldots \alpha_{s}\right)}+\alpha R \varphi_{\alpha_{1} \ldots \alpha_{s}},
$$

and those to the gauge identity generators are

$$
\begin{aligned}
\Delta \tilde{\mathcal{J}}_{1, \alpha_{1} \ldots \alpha_{s-1}}^{\mu_{1} \ldots \mu_{s-1}}= & \left.\left.-(s-1)\left[(s-2) \delta_{\rho \sigma\left(\alpha_{1} \ldots \alpha_{s-3}\right.}^{\mu_{1} \ldots \mu_{s-1}} R_{\alpha_{s-2}}^{\rho}{ }^{\sigma} \alpha_{s-1}\right)-\delta_{\rho\left(\alpha_{1} \ldots \alpha_{s-2}\right.}^{\mu_{1} \ldots \mu_{s-1}} R^{\rho}{ }_{\left.\alpha_{s-1}\right)}\right)\right] \\
& -\alpha R \delta_{\alpha_{1} \ldots \alpha_{s-1}}^{\mu_{1} \ldots \mu_{s-1}}, \\
\Delta \tilde{\mathcal{K}}_{1, \alpha_{1} \ldots \alpha_{s-1}}^{\mu_{1} \ldots \mu_{s-2}}= & -\frac{(s-1)(s-2)}{D-2}\left[Y_{\left(\alpha_{1} \alpha_{2}\right.}{ }^{\left(\mu_{1}\right.} \delta_{\left.\alpha_{3} \ldots \alpha_{s-1}\right)}^{\left.\mu_{2} \ldots \mu_{s-2}\right)}+\frac{4 D-7}{3 D+6} Z_{\left(\alpha_{1} \alpha_{2}\right.}{ }^{\left(\mu_{1}\right.} \delta_{\left.\alpha_{3} \ldots \alpha_{s-1}\right)}^{\left.\mu_{2} \ldots \mu_{s-2}\right)}\right] \\
& -\frac{2(s-1)}{D-1}\left[\frac{s-1}{D+2} \nabla_{\left(\alpha_{1}\right.} R \delta_{\left.\alpha_{2} \ldots \alpha_{s-1}\right)}^{\mu_{1} \ldots \mu_{s-1}}+\frac{s-2}{D-2} g_{\left(\alpha_{1} \alpha_{2}\right.} \nabla^{\left(\mu_{1}\right.} R \delta_{\left.\alpha_{3} \ldots \alpha_{s-1}\right)}^{\left.\mu_{2} \ldots \mu_{s-2}\right)}\right],
\end{aligned}
$$

while the remaining anomalous terms $\mathcal{B}_{\alpha_{1} \ldots \alpha_{s-1}}$ read

$$
\begin{aligned}
\mathcal{B}_{\alpha_{1} \ldots \alpha_{s-1}}= & -\frac{(s-1)(s-2)}{D-2}\left[(D-2) X^{\mu \nu \rho}{ }_{\left(\alpha_{1} \alpha_{2}\right.} \varphi_{\left.\alpha_{3} \ldots \alpha_{s-1}\right) \mu \nu \rho}+Y^{\mu \nu \rho} g_{\left(\alpha_{1} \alpha_{2}\right.} \varphi_{\left.\alpha_{3} \ldots \alpha_{s-1}\right) \mu \nu \rho}\right] \\
& +\left(\frac{s-1}{D-2}\right)\left[(2 s+D-6) Y^{\mu \nu}{ }_{\left(\alpha_{1}\right.} \varphi_{\left.\alpha_{2} \ldots \alpha_{s-1}\right) \mu \nu}-\left(\frac{s+2 D-6}{3}\right) Z^{\mu \nu}{ }_{\left(\alpha_{1}\right.} \varphi_{\left.\alpha_{2} \ldots \alpha_{s-1}\right) \mu \nu}\right] \\
& +\left[\frac{2(s-1)(s+D-2)}{(D-1)(D+2)}-\alpha\right]\left(\nabla^{\mu} R\right) \varphi_{\alpha_{1} \ldots \alpha_{s-1} \mu}
\end{aligned}
$$

where $X_{\mu \nu \rho}^{\alpha \beta}, Y_{\mu \nu \rho}$ and $Z_{\mu \nu \rho}$ are the irreducible Lorentz tensors defined in eqs. (1.1)-(1.3). These problematic terms vanish if the gravitational background satisfy, for generic spin, the 
conditions (1.1)-(1.3) plus the condition (2.19). The first gauge identity, $\mathcal{G}_{1, \alpha_{1} \ldots \alpha_{s-1}}=0$, is then fulfilled with the corrected equations:

$$
\begin{aligned}
\Delta I_{\mu_{1} \ldots \mu_{s}} & =\Delta \tilde{I}_{\mu_{1} \ldots \mu_{s}}+\mathcal{O}\left(R^{2}\right), \\
\Delta J_{\mu_{1} \ldots \mu_{s-1}} & =\mathcal{O}\left(R^{2}\right), \\
\Delta K_{\mu_{1} \ldots \mu_{s-2}} & =\mathcal{O}\left(R^{2}\right),
\end{aligned}
$$

and the corrected gauge identity generators:

$$
\begin{aligned}
\Delta \mathcal{I}_{1, \alpha_{1} \ldots \alpha_{s-1}}^{\mu_{1} \ldots \mu_{s}} & =\mathcal{O}\left(R^{2}\right), \\
\Delta \mathcal{J}_{1, \alpha_{1} \ldots \alpha_{s-1}}^{\mu_{1} \ldots \mu_{s-1}} & =\Delta \tilde{\mathcal{J}}_{1, \alpha_{1} \ldots \alpha_{s-1}}^{\mu_{1} \ldots \mu_{s-1}}+\mathcal{O}\left(R^{2}\right), \\
\Delta \mathcal{K}_{1, \alpha_{1} \ldots \alpha_{s-1}}^{\mu_{1} \ldots \mu_{s-2}} & =\Delta \tilde{\mathcal{K}}_{1, \alpha_{1} \ldots \alpha_{s-1}}^{\mu_{1} \ldots \mu_{s-2}}+\mathcal{O}\left(R^{2}\right) .
\end{aligned}
$$

On the other hand, corresponding to $i=1$ and $i=2$ respectively, the second and third gauge identities call for

$$
\Delta \mathcal{K}_{2, \alpha_{1} \ldots \alpha_{s-2}}^{\mu_{1} \ldots \mu_{s-2}}=-\alpha R \delta_{\alpha_{1} \ldots \alpha_{s-2}}^{\mu_{1} \ldots \mu_{s-2}}+\mathcal{O}\left(R^{2}\right)
$$

with all other corrections being only $\mathcal{O}\left(R^{2}\right)$.

Open Access. This article is distributed under the terms of the Creative Commons Attribution License (CC-BY 4.0), which permits any use, distribution and reproduction in any medium, provided the original author(s) and source are credited.

\section{References}

[1] S. Weinberg, Photons and gravitons in $S$ matrix theory: derivation of charge conservation and equality of gravitational and inertial mass, Phys. Rev. 135 (1964) B1049 [INSPIRE].

[2] M.T. Grisaru and H.N. Pendleton, Soft spin-3/2 fermions require gravity and supersymmetry, Phys. Lett. B 67 (1977) 323 [INSPIRE].

[3] M.T. Grisaru, H.N. Pendleton and P. van Nieuwenhuizen, Supergravity and the S matrix, Phys. Rev. D 15 (1977) 996 [INSPIRE].

[4] C. Aragone and S. Deser, Consistency problems of hypergravity, Phys. Lett. B 86 (1979) 161 [INSPIRE].

[5] S. Deser and Z. Yang, Inconsistency of spin-4-spin-2 gauge field couplings, Class. Quant. Grav. 7 (1990) 1491 [INSPIRE].

[6] S. Weinberg and E. Witten, Limits on massless particles, Phys. Lett. B 96 (1980) 59 [INSPIRE].

[7] M. Porrati, Universal limits on massless high-spin particles, Phys. Rev. D 78 (2008) 065016 [arXiv: 0804.4672] [INSPIRE].

[8] M. Fierz and W. Pauli, On relativistic wave equations for particles of arbitrary spin in an electromagnetic field, Proc. Roy. Soc. Lond. A 173 (1939) 211 [InSPIRE].

[9] G. Velo and D. Zwanziger, Propagation and quantization of Rarita-Schwinger waves in an external electromagnetic potential, Phys. Rev. 186 (1969) 1337 [INSPIRE]. 
[10] G. Velo and D. Zwanziger, Noncausality and other defects of interaction Lagrangians for particles with spin one and higher, Phys. Rev. 188 (1969) 2218 [INSPIRE].

[11] G. Velo, Anomalous behaviour of a massive spin two charged particle in an external electromagnetic field, Nucl. Phys. B 43 (1972) 389 [InSPIRE].

[12] A. Shamaly and A.Z. Capri, Propagation of interacting fields, Annals Phys. 74 (1972) 503 [INSPIRE].

[13] M. Hortacsu, Demonstration of noncausality for the Rarita-Schwinger equation, Phys. Rev. D 9 (1974) 928 [INSPIRE].

[14] M. Kobayashi and A. Shamaly, Minimal electromagnetic coupling for massive spin-2 fields, Phys. Rev. D 17 (1978) 2179 [inSPIRE].

[15] M. Kobayashi and A. Shamaly, The tenth constraint in the minimally coupled spin-2 wave equations, Prog. Theor. Phys. 61 (1979) 656 [INSPIRE].

[16] S. Deser, V. Pascalutsa and A. Waldron, Massive spin-3/2 electrodynamics, Phys. Rev. D 62 (2000) 105031 [hep-th/0003011] [INSPIRE].

[17] S. Deser and A. Waldron, Inconsistencies of massive charged gravitating higher spins, Nucl. Phys. B 631 (2002) 369 [hep-th/0112182] [INSPIRE].

[18] P.C. Argyres and C.R. Nappi, Massive spin-2 bosonic string states in an electromagnetic background, Phys. Lett. B 224 (1989) 89 [INSPIRE].

[19] M. Porrati, R. Rahman and A. Sagnotti, String theory and the Velo-Zwanziger problem, Nucl. Phys. B 846 (2011) 250 [arXiv:1011.6411] [INSPIRE].

[20] M. Porrati and R. Rahman, Causal propagation of a charged spin-3/2 field in an external electromagnetic background, Phys. Rev. D 80 (2009) 025009 [arXiv:0906.1432] [INSPIRE].

[21] I.L. Buchbinder, T.V. Snegirev and Y. Zinoviev, Cubic interaction vertex of higher-spin fields with external electromagnetic field, Nucl. Phys. B 864 (2012) 694 [arXiv:1204.2341] [inSPIRE].

[22] I.L. Buchbinder, D.M. Gitman and V.D. Pershin, Causality of massive spin-2 field in external gravity, Phys. Lett. B 492 (2000) 161 [hep-th/0006144] [INSPIRE].

[23] I.L. Buchbinder, V.A. Krykhtin and A.A. Reshetnyak, BRST approach to Lagrangian construction for fermionic higher spin fields in (A)dS space, Nucl. Phys. B 787 (2007) 211 [hep-th/0703049] [INSPIRE].

[24] I.L. Buchbinder and V.A. Krykhtin, Progress in gauge invariant Lagrangian construction for massive higher spin fields, submitted to JHEP (2007) [arXiv:0710.5715] [INSPIRE].

[25] I.L. Buchbinder, V.A. Krykhtin and P.M. Lavrov, Gauge invariant Lagrangian formulation of higher spin massive bosonic field theory in AdS space, Nucl. Phys. B 762 (2007) 344 [hep-th/0608005] [INSPIRE].

[26] I.L. Buchbinder, V.A. Krykhtin and P.M. Lavrov, On manifolds admitting the consistent Lagrangian formulation for higher spin fields, Mod. Phys. Lett. A 26 (2011) 1183 [arXiv: 1101.4860] [INSPIRE].

[27] Y. Zinoviev, On massive high spin particles in AdS, hep-th/0108192 [INSPIRE].

[28] Y. Zinoviev, On massive spin-2 interactions, Nucl. Phys. B 770 (2007) 83 [hep-th/0609170] [INSPIRE]. 
[29] Y. Zinoviev, On massive spin-2 electromagnetic interactions, Nucl. Phys. B 821 (2009) 431 [arXiv:0901.3462] [INSPIRE].

[30] K. Skenderis and P.K. Townsend, Pseudo-supersymmetry and the domain-wall/cosmology correspondence, J. Phys. A 40 (2007) 6733 [hep-th/0610253] [InSPIRE].

[31] L. Randall and R. Sundrum, An alternative to compactification, Phys. Rev. Lett. 83 (1999) 4690 [hep-th/9906064] [INSPIRE].

[32] O. DeWolfe, D.Z. Freedman, S.S. Gubser and A. Karch, Modeling the fifth-dimension with scalars and gravity, Phys. Rev. D 62 (2000) 046008 [hep-th/9909134] [INSPIRE].

[33] C. Csáki, TASI lectures on extra dimensions and branes, in From fields to strings, vol. 2, M. Shifman et al. eds., World Scientific, Singapore (2005), pg. 967 [hep-ph/0404096] [INSPIRE].

[34] V. Dzhunushaliev, V. Folomeev and M. Minamitsuji, Thick brane solutions, Rept. Prog. Phys. 73 (2010) 066901 [arXiv: 0904.1775] [INSPIRE].

[35] W.M. Seiler, Involution: the formal theory of differential equations and its applications in computer algebra, in Algorithms and computations in mathematics, vol. 24, Springer-Verlag, Berlin Heidelberg Germany (2010).

[36] D.S. Kaparulin, S.L. Lyakhovich and A.A. Sharapov, Consistent interactions and involution, JHEP 01 (2013) 097 [arXiv: 1210.6821] [INSPIRE].

[37] A. Einstein, The meaning of the relativity, $5^{\text {th }}$ ed., Princeton University Press, Princeton U.S.A. (1955).

[38] I. Cortese, R. Rahman and M. Sivakumar, Consistent non-minimal couplings of massive higher-spin particles, Nucl. Phys. B 879 (2014) 143 [arXiv:1307.7710] [InSPIRE].

[39] E.S. Fradkin and A.A. Tseytlin, Conformal supergravity, Phys. Rept. 119 (1985) 233 [INSPIRE].

[40] E.S. Fradkin and V.Y. Linetsky, Superconformal higher spin theory in the cubic approximation, Nucl. Phys. B 350 (1991) 274 [INSPIRE].

[41] A.Y. Segal, Conformal higher spin theory, Nucl. Phys. B 664 (2003) 59 [hep-th/0207212] [INSPIRE].

[42] A.A. Tseytlin, On partition function and Weyl anomaly of conformal higher spin fields, Nucl. Phys. B 877 (2013) 598 [arXiv: 1309.0785] [INSPIRE].

[43] A.A. Tseytlin, Weyl anomaly of conformal higher spins on six-sphere, Nucl. Phys. B 877 (2013) 632 [arXiv:1310.1795] [INSPIRE].

[44] M. Beccaria, X. Bekaert and A.A. Tseytlin, Partition function of free conformal higher spin theory, JHEP 08 (2014) 113 [arXiv: 1406.3542] [INSPIRE].

[45] R.R. Metsaev, CFT adapted approach to massless fermionic fields, AdS/CFT and fermionic conformal fields, arXiv:1311.7350 [INSPIRE].

[46] R.R. Metsaev, Light-cone gauge approach to arbitrary spin fields, currents and shadows, J. Phys. A 47 (2014) 375401 [arXiv:1312.5679] [INSPIRE].

[47] R.R. Metsaev, Arbitrary spin conformal fields in (A)dS, Nucl. Phys. B 885 (2014) 734 [arXiv: 1404.3712] [INSPIRE]. 
[48] R.R. Metsaev, BRST invariant effective action of shadow fields, conformal fields and $A d S / C F T$, arXiv:1407.2601 [INSPIRE].

[49] A.O. Barvinsky, Thermal power spectrum in the CFT driven cosmology, JCAP 10 (2013) 059 [arXiv: 1308.4451] [INSPIRE].

[50] J.S. Dowker, Numerical evaluation of spherical GJMS determinants for even dimensions, arXiv: 1310.0759 [INSPIRE].

[51] S. Yokoyama, A note on large- $N$ thermal free energy in supersymmetric Chern-Simons vector models, JHEP 01 (2014) 148 [arXiv:1310.0902] [INSPIRE].

[52] B.E.W. Nilsson, Towards an exact frame formulation of conformal higher spins in three dimensions, arXiv:1312.5883 [INSPIRE].

[53] S. Giombi, I.R. Klebanov and B.R. Safdi, Higher spin $A d S_{d+1} / C F T_{d}$ at one loop, Phys. Rev. D 89 (2014) 084004 [arXiv:1401.0825] [INSPIRE].

[54] M.A. Vasiliev, Higher-spin theory and space-time metamorphoses, arXiv:1404.1948 [INSPIRE].

[55] T. Nutma and M. Taronna, On conformal higher spin wave operators, JHEP 06 (2014) 066 [arXiv: 1404.7452] [INSPIRE].

[56] R. Aros, F. Bugini and D.E. Diaz, On Rényi entropy for free conformal fields: holographic and q-analog recipes, arXiv:1408.1931 [INSPIRE].

[57] M. Gremm, Four-dimensional gravity on a thick domain wall, Phys. Lett. B 478 (2000) 434 [hep-th/9912060] [INSPIRE].

[58] M. Gremm, Thick domain walls and singular spaces, Phys. Rev. D 62 (2000) 044017 [hep-th/0002040] [INSPIRE].

[59] C. Germani and A. Kehagias, Higher-spin fields in braneworlds, Nucl. Phys. B 725 (2005) 15 [hep-th/0411269] [INSPIRE].

[60] C. Germani and A. Schelpe, Interactions of higher spin fields with gravity and branes in $A d S_{5}$, Phys. Rev. D 78 (2008) 036010 [arXiv:0712.2243] [INSPIRE].

[61] S. Ferrara and M. Porrati, 4D localization in Randall-Sundrum 2 supergravity and in Vasiliev theories, Phys. Lett. B 704 (2011) 249 [arXiv:1108.1993] [INSPIRE].

[62] C. Csáki, J. Erlich, T.J. Hollowood and Y. Shirman, Universal aspects of gravity localized on thick branes, Nucl. Phys. B 581 (2000) 309 [hep-th/0001033] [INSPIRE].

[63] R.R. Metsaev, Massless mixed symmetry bosonic free fields in d-dimensional anti-de Sitter space-time, Phys. Lett. B 354 (1995) 78 [INSPIRE].

[64] R.R. Metsaev, Arbitrary spin massless bosonic fields in d-dimensional anti-de Sitter space, Lect. Notes Phys. 524 (1999) 331 [hep-th/9810231] [INSPIRE].

[65] R.R. Metsaev, Massive totally symmetric fields in AdS $S_{d}$, Phys. Lett. B 590 (2004) 95 [hep-th/0312297] [INSPIRE].

[66] D.J. Kapner et al., Tests of the gravitational inverse-square law below the dark-energy length scale, Phys. Rev. Lett. 98 (2007) 021101 [hep-ph/0611184] [INSPIRE].

[67] P. Breitenlohner and D.Z. Freedman, Positive energy in anti-de Sitter backgrounds and gauged extended supergravity, Phys. Lett. B 115 (1982) 197 [INSPIRE].

[68] M. Porrati, Old and new no go theorems on interacting massless particles in flat space, arXiv:1209.4876 [INSPIRE]. 
[69] S. Deser and A. Waldron, Arbitrary spin representations in de Sitter from dS/CFT with applications to dS supergravity, Nucl. Phys. B 662 (2003) 379 [hep-th/0301068] [INSPIRE].

[70] C.M. Bender and T.T. Wu, Anharmonic oscillator. 2: a study of perturbation theory in large order, Phys. Rev. D 7 (1973) 1620 [InSPIRE].

[71] S.L. Lyakhovich, A.Y. Segal and A.A. Sharapov, A universal model of $D=4$ spinning particle, Phys. Rev. D 54 (1996) 5223 [hep-th/9603174] [INSPIRE].

[72] S.L. Lyakhovich, A.A. Sharapov and K.M. Shekhter, Spinning particle dynamics on six-dimensional Minkowski space, J. Math. Phys. 38 (1997) 4086 [hep-th/9609133] [INSPIRE].

[73] S.L. Lyakhovich, A.A. Sharapov and K.M. Shekhter, A uniform model of the massive spinning particle in any dimension, Int. J. Mod. Phys. A 15 (2000) 4287 [hep-th/0002247] [INSPIRE].

[74] K.B. Alkalaev and S.L. Lyakhovich, On the consistency problem of interactions of $(2+1)$ massive spinning particle, Mod. Phys. Lett. A 14 (1999) 2727 [INSPIRE].

[75] K. Hallowell and A. Waldron, The symmetric tensor Lichnerowicz algebra and a novel associative Fourier-Jacobi algebra, SIGMA 3 (2007) 089 [arXiv:0707.3164] [INSPIRE].

[76] K. Hallowell and A. Waldron, Supersymmetric quantum mechanics and super-Lichnerowicz algebras, Commun. Math. Phys. 278 (2008) 775 [hep-th/0702033] [INSPIRE].

[77] F. Bastianelli, O. Corradini and E. Latini, Spinning particles and higher spin fields on (A)dS backgrounds, JHEP 11 (2008) 054 [arXiv:0810.0188] [INSPIRE].

[78] O. Corradini, Half-integer higher spin fields in (A)dS from spinning particle models, JHEP 09 (2010) 113 [arXiv: 1006.4452] [INSPIRE].

[79] F. Bastianelli, R. Bonezzi, O. Corradini and E. Latini, Massive and massless higher spinning particles in odd dimensions, JHEP 09 (2014) 158 [arXiv:1407.4950] [INSPIRE].

[80] M. Asorey and D. Garcia-Alvarez, Higher spin dark matter, AIP Conf. Proc. 1241 (2010) 1192 [INSPIRE].

[81] S. Deser, E. Joung and A. Waldron, Gravitational- and self-coupling of partially massless spin-2, Phys. Rev. D 86 (2012) 104004 [arXiv:1301.4181] [INSPIRE].

[82] S. Deser, E. Joung and A. Waldron, Partial masslessness and conformal gravity, J. Phys. A 46 (2013) 214019 [arXiv:1208.1307] [INSPIRE].

[83] E. Joung, L. Lopez and M. Taronna, On the cubic interactions of massive and partially-massless higher spins in (A)dS, JHEP 07 (2012) 041 [arXiv:1203.6578] [INSPIRE].

[84] E. Joung, L. Lopez and M. Taronna, Generating functions of (partially-)massless higher-spin cubic interactions, JHEP 01 (2013) 168 [arXiv:1211.5912] [INSPIRE].

[85] E. Joung, W. Li and M. Taronna, No-go theorems for unitary and interacting partially massless spin-two fields, Phys. Rev. Lett. 113 (2014) 091101 [arXiv:1406.2335] [INSPIRE].

[86] S. Deser, M. Sandora and A. Waldron, Nonlinear partially massless from massive gravity?, Phys. Rev. D 87 (2013) 101501 [arXiv:1301.5621] [INSPIRE].

[87] C. de Rham, K. Hinterbichler, R.A. Rosen and A.J. Tolley, Evidence for and obstructions to nonlinear partially massless gravity, Phys. Rev. D 88 (2013) 024003 [arXiv:1302.0025] [INSPIRE]. 